\title{
COMPOSICIÓN Y ABUNDANCIA DE LAS PTERIDOFITAS EN EL BOSQUE MESÓFILO DE MONTAÑA DEL MUNICIPIO DE Tlanchinol, Hidalgo, MÉxico
}

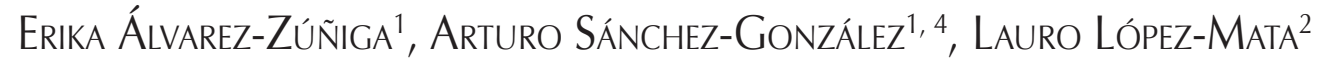 \\ y José Daniel Tejero-Díez ${ }^{3}$ \\ ${ }^{1}$ Centro de Investigaciones Biológicas, Universidad Autónoma del Estado de Hidalgo, Hidalgo, México \\ ${ }^{2}$ Programa de Botánica, Colegio de Postgraduados \\ ${ }^{3}$ Facultad de Estudios Superiores Iztacala, Universidad Nacional Autónoma de México \\ ${ }^{4}$ Autor para la correspondencia: arturosg@uaeh.edu.mx
}

\begin{abstract}
Resumen: El conocimiento sobre la riqueza y distribución de las especies de helechos y licopodios en el estado de Hidalgo, es escaso por lo que el objetivo principal de esta investigación fue realizar un inventario de las especies de Pteridofitas, conocer sus patrones de abundancia y los sustratos de crecimiento en el bosque mesófilo de montaña del municipio de Tlanchinol, Hidalgo. El estudio florístico se basó en la recolecta exhaustiva de ejemplares en 30 sitios y en la consulta de especímenes en los herbarios ENCB, FCME, HGOM, IZTA, MEXU y XAL. Para estimar la abundancia de las especies se establecieron 24 parcelas de muestreo de $400 \mathrm{~m}^{2}$ cada una. Se identificaron 21 familias, 53 géneros, 130 especies y diez variedades. La completitud del inventario osciló entre 73.4 y $87 \%$, de acuerdo con los estimadores de riqueza de especies Clench y ACE, respectivamente. Sólo el 19\% de las especies presentó abundancias promedio mayores a cinco individuos $/ 400 \mathrm{~m}^{2}$. La mayoría de las especies se desarrollan sobre suelo (58.1\%) o son de hábito epifito (25.5\%). Además, se citan 12 especies como nuevos registros para el estado de Hidalgo y cinco taxones que se encuentran en alguna categoría de riesgo dentro de la Norma Oficial Mexicana NOM-059-SEMARNAT-2010.
\end{abstract}

Palabras clave: bosque de montaña, helechos, licopodios, riqueza de especies.

\begin{abstract}
The knowledge on the species richness and distribution of Pteridophytes in the state of Hidalgo is scarce, so that the objective of this research was to carry out an inventory of Pteridophytes to get insights on its patterns of abundance and substrates of growth in the cloud forest of Tlanchinol, Hidalgo. The floristic study was based on an exhaustive field recollection of specimens from 30 localities as well as vouchers review at ENCB, FCME, HGOM, IZTA, MEXU, and XAL herbaria. Species abundances were estimated from sampling of 24 plots $400 \mathrm{~m}^{2}$ each. In total 21 families, 53 genera, 130 species, and ten varieties were identified. The inventory completeness ranged from 73.4 to $87 \%$, after Clench and ACE estimators, respectively. Only $19 \%$ of the species had abundances greater than five individuals $/ 400 \mathrm{~m}^{2}$. Most species have a terrestrial life form, growing on forest floor $(58.1 \%)$ or are epiphytes (25.5\%). Twelve species are new records for the state of Hidalgo and five species are under protection by the Mexican Official Norm NOM-059-SEMARNAT-2010.
\end{abstract}

Key words: ferns, lycophytes, mountain forest, species richness.

L os helechos y licopodios (Pteridofitas) son plantas vasculares que representan dos líneas filogenéticas divergentes. Sin embargo, comparten el mismo tipo de ciclo de vida, el cual se caracteriza por presentar dos fases alternantes independientes: la fase dominante es la esporófita, productora de esporas que diseminan el material genético producto de la meiosis y la fase gametofítica, incospicua, es donde se desarrollan los gametos. Los helechos y licopodios son plantas herbáceas, perennes (con alguna excepción), carecen de crecimiento secundario (Moran, 2004), presentan una gran variedad de tamaños, formas de crecimiento y se localizan en casi todos los tipos de vegetación en intervalos de altitud de entre 0 y 5,000 m; algunas especies tienen distribución cosmopolita (Kessler, 2010).

En México la mayor riqueza de especies de helechos y licopodios se encuentra en la zona ecológica templada húmeda (Tejero-Díez et al., 2011), que incluye al bosque mesófilo de montaña (BMM) cuya riqueza florística por unidad de área, es la más elevada de todos los tipos de vegetación de México (Rzedowski, 1978; Villaseñor, 2010). En este tipo 
de vegetación, los helechos y licopodios son un componente estructural importante en el sotobosque, donde desempeñan funciones ecológicas relevantes (Riba, 1998; Mehltreter, 2008).

El estado de Hidalgo ocupa el tercer lugar en México con mayor superficie de BMM $(21,641$ ha), el segundo es Chiapas con 27,526 ha y Oaxaca en primer sitio con 35,217 ha (Ortega-Escalona y Castillo-Campos, 1996; Luna-Vega et al., 2000). Existen varios estudios sobre la flora vascular de los BMM de la entidad, en los cuales se incluyen inventarios parciales de helechos y licopodios: destaca el realizado en el municipio de Tlanchinol donde Luna-Vega et al. (1994) registran 43 especies; el del municipio de Tenango de Doria donde Alcántara-Ayala y Luna-Vega (1997) mencionan 39 especies; en Molocotlán, Molango-Xochicoatlán, MayorgaSaucedo et al. (1998) encontraron 32 especies; en Eloxochitlán y Tlahuelompa se enlistan 31 especies (Alcántara-Ayala y Luna-Vega, 2001) y en Monte Grande, Lolotla Ponce-Vargas et al. (2006) registraron 32 especies.

Recientemente en distintas regiones y tipos de vegetación del estado de Hidalgo, se han realizado inventarios florísticos, exclusivamente de helechos y licopodios, que indican que la riqueza de especies en ambos grupos de plantas, es más elevada de lo que se había mencionado en estudios previos. Por ejemplo, en la Reserva de la Biosfera Barranca de Metztitlán (RBBM), Cuevas-Hernández (2008) encontró 76 especies; en el Parque Nacional Los Mármoles (PNM) Ramírez-Cruz et al. (2009) mencionan 71 especies y en el Parque Nacional El Chico (PNC) Serrano-Martínez (2010) identificó 62 especies. En los municipios con BMM de la entidad, en particular, se ha constatado que la riqueza de especies de Pteridofitas es todavía más alta, lo que se relaciona con los requerimientos ambientales (principalmente de humedad) de estos organismos: en el municipio de Tenango de Doria, Zúñiga-Salvatierra (2009) encontró 107 especies, en el de Calnali, Pérez-Cervantes (2009) registró 115 especies y en el de Zacualtipán de Ángeles, Pérez-Paredes et al. (2012) mencionan 125 especies.

De acuerdo con Luna-Vega et al. (1994, 2000) y León y Paniagua et al. (2010), el BMM del municipio de Tlanchinol, representa un área que debería de ser considerada prioritaria para la conservación por su elevada riqueza de especies de plantas y la presencia de elementos endémicos, por lo que es necesario frenar el disturbio provocado por las actividades humanas. Tomando en cuenta que sólo existe un artículo científico publicado sobre la flora del municipio de Tlanchinol, que incluye un inventario parcial de helechos y licopodios (Luna-Vega et al., 1994) y que los estudios recientes realizados exclusivamente con Pteridofitas en los BMM del estado de Hidalgo, resaltan la presencia de un número elevado de especies; se planteó como hipótesis de trabajo que la composición taxonómica de los helechos y licopodios del BMM del municipio de Tlanchinol es mucho más elevada de lo que hasta ahora se conoce. Por ello, los objetivos del presente estudio fueron: (1) contribuir al conocimiento florístico del BMM en el municipio de Tlanchinol, estado de Hidalgo, mediante el estudio de los helechos y licopodios y (2) aportar información sobre su abundancia, hábitos de crecimiento y amplitud de distribución en este tipo de vegetación.

\section{Materiales y métodos}

Zona de estudio. El municipio de Tlanchinol se localiza en la porción Norte del estado de Hidalgo, limita al Norte con el estado de San Luis Potosí, al Sur con el municipio de Calnali, al Oeste con el municipio de Lolotla y al Este con los municipios de Huazalingo y Huejutla. Ocupa una superficie de aproximadamente $380 \mathrm{~km}^{2}$, que representa el $1.9 \%$ de la superficie estatal (Figura 1). Geográficamente se encuentra entre los paralelos $21^{\circ} 00^{\prime}$ y $21^{\circ} 10^{\prime}$ de latitud Norte y $98^{\circ}$ $30^{\prime}$ y $98^{\circ} 35^{\prime}$ de longitud Oeste, en una altitud promedio de 1,590 m (SEGOB, 1988; INEGI, 1996).

Suelos. Son derivados de rocas del Terciario, Cuaternario y Mesozoico, arcillosos y en un 60\% de tipo litosol (SEGOB, 1988; INEGI, 1992).

Orografía. La superficie es abrupta en gran parte del municipio, con muchas barrancas, además de tener mesetas y valles, al Este presenta una planicie y al Oeste llanos con pendientes mayores a 10\% (INEGI, 1996).

Clima. Predomina el clima templado, con temperatura promedio de $17.2{ }^{\circ} \mathrm{C}$ y precipitación pluvial de 2,156.2 mm por año, con período de lluvias principalmente en verano, de mayo a septiembre y menor proporción en invierno (SEGOB, 1988; INEGI, 2004; Pavón-Hernández y Meza-Sánchez, 2009).

Hidrología. Se encuentra dentro de la región del río Pánuco y la cuenca del río Moctezuma; cruzan al municipio los ríos Amajac, Quetzaltongo, Santa María, Tehuetlán y Xalpan (INEGI, 1996).

Vegetación. Se encuentra cubierto por masas forestales de BMM interrumpidas por terreno agropecuario. De acuerdo con Luna-Vega et al. (1994), Puig (1976) y VillavicencioNieto y Pérez-Escandón (2005) en el municipio de Tlanchinol las especies arbóreas comunes son: Alnus acuminata, Bejaria aestuans, Clethra mexicana, Dalbergia palo-escrito, Liquidambar styraciflua, Magnolia schiedeana, Pinus patula, Quercus eugenifolia y $Q$. sartorii; en el estrato arbustivo sobresalen Bocconia frutescens, Conostegia xalapensis, Decatropis bicolor y Miconia oligotricha, entre otras. Asimismo se registra la presencia de helechos arborescentes del género Cyathea.

Trabajo en campo. Se realizaron ocho salidas de campo, entre marzo de 2009 y septiembre de 2010, en este lapso el trabajo en campo se dividió en dos actividades: (1) exploración y recolecta de ejemplares en 30 localidades y (2) estimación de la densidad de individuos por especie por medio 


\section{LAS PTERIDOFITAS DE TLANCHINOL}

de unidades de muestreo de $400 \mathrm{~m}^{2}$ (cuadros de $20 \times 20 \mathrm{~m}$ ) cada una, en 24 localidades representativas de los cambios físicos del ambiente y/o de la dominancia de las especies arbóreas del total de las localidades exploradas (Figura 1). Las localidades se pre-seleccionaron mediante cartas topográficas escala 1:50,000 y en el campo, el criterio final de selección de cada localidad fue con base en la altitud (para lo cual se utilizó un geo-posicionador), el tipo de vegetación $(\mathrm{BMM})$ y la cobertura del dosel $>50 \%$, verificada con un densitómetro esférico. El intervalo altitudinal donde se realizó el trabajo fluctuó entre 1,107 y 1,903 m. Para el muestreo de la Pteridoflora se seleccionaron sólo 24 de las 30 localidades donde previamente se llevó a cabo la recolección de ejemplares, por la elevada cantidad de tiempo que requiere el conteo de los individuos de cada especie; el tamaño de las parcelas de muestreo es el área mínima sugerida para el estudio comparativo de las Pteridofitas en bosques tropicales húmedos (Kessler, 2002; Watkins et al., 2006). Las especies epifitas que se encontraban en las partes altas de los árboles se recolectaron mediante el uso de una garrocha o buscando árboles o ramas recientemente caídas; el conteo de los individuos cuando fue necesario, se realizó con binoculares (Kessler, 2002). Para la estimación de la densidad de individuos por especie, cada uno de los tallos aéreos se consideró como un individuo, independientemen- te de que fueran o no clones (Watkins et al., 2006). Debido a la complejidad y ambigüedad en la definición del BMM, el criterio para su identificación se basó principalmente en el reconocimiento de especies arbóreas y arbustivas típicas de este tipo de vegetación, en la abundancia de especies epifitas y en las condiciones físicas de humedad elevada y temperatura moderada en el sotobosque (Luna-Vega et al., 1994; Villaseñor, 2010).

Trabajo de laboratorio. Para la determinación de las especies se utilizaron las claves dicotómicas de la obra de Mickel y Smith (2004) y en el caso del género Elaphoglossum se revisó el trabajo de Rojas-Alvarado (2003). Los ejemplares herborizados se incorporaron a la colección de los herbarios HGOM y MEXU. Además se revisaron las colecciones de los herbarios ENCB (Escuela Nacional de Ciencias Biológicas), FCME (Facultad de Ciencias, Universidad Nacional Autónoma de México), HGOM (Centro de Investigaciones Biológicas), IZTA (Facultad de Estudios Superiores Iztacala, Universidad Nacional Autónoma de México), MEXU (Instituto de Biología, Universidad Nacional Autónoma de México) y XAL (Instituto de Ecología, A.C.); y los listados florísticos de los estudios regionales enumerados en los antecedentes, con el objetivo de incorporar en el inventario a las especies no observadas en el presente trabajo.

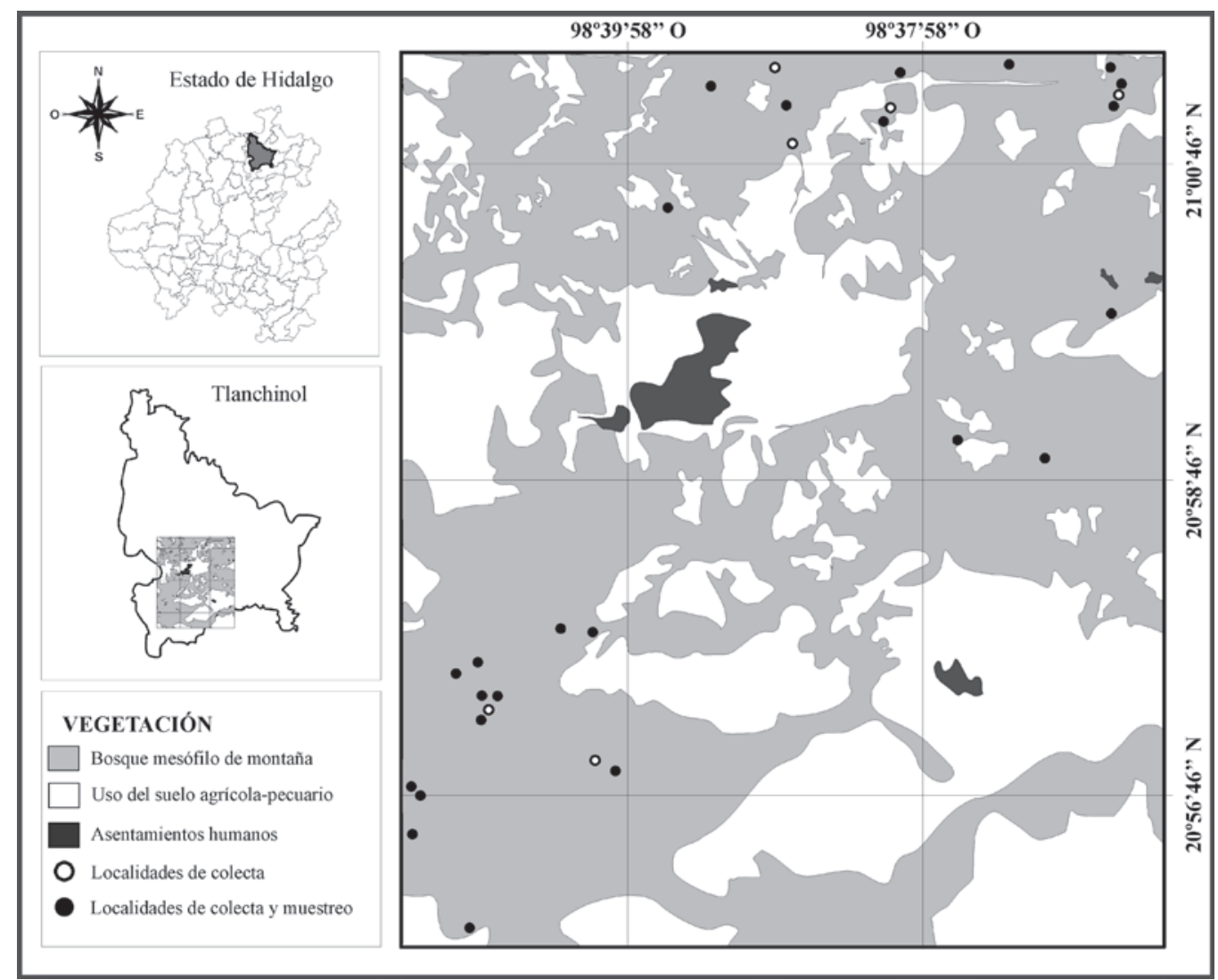

Figura 1. Localización geográfica del municipio de Tlanchinol, estado de Hidalgo y posición de los sitios de colecta y muestreo 
La amplitud de distribución de cada una de las especies en el área de estudio, se estimó por medio de su frecuencia relativa en las 30 localidades de recolecta (Magurran, 2004). El número promedio de individuos por especie en $400 \mathrm{~m}^{2}$, se calculó dividiendo el número total de individuos de cada especie entre el número total de parcelas de muestreo (24 parcelas, Apéndice 1). El porcentaje de completitud del inventario de especies se evaluó con los datos de abundancia de cada especie, utilizando el estimador no paramétrico ACE y el modelo asintótico de Clench (Jiménez-Valverde y Hortal, 2003). Los análisis se realizaron con los programas de cálculo: EstimateS, versión 8.2 (Colwell, 2009) y STATISTICA (StatSoft Inc., 2004).

Además, se comparó la riqueza de especies del municipio de Tlanchinol, con la de otros tres municipios (Calnali, Tenango de Doria y Zacualtipán de Ángeles) y tres áreas naturales protegidas (PNC, PNM y RBBM) del estado de Hidalgo, que contaban con estudios florísticos enfocados exclusivamente a las Pteridofitas, utilizando el índice de biodiversidad taxonómica (IB): $\mathrm{IB}=\mathrm{S} / \mathrm{Ln} \mathrm{A}$, donde $\mathrm{S}=$ número de especies registradas y $\mathrm{LnA}=$ logaritmo natural del tamaño del área (Ponce et al., 2002; Ramírez-Cruz et al., 2009).

\section{Resultados}

En el BMM del municipio de Tlanchinol se recolectaron y determinaron 416 ejemplares de helechos y licopodios, los cuales correspondieron, de acuerdo al sistema de clasificación de Smith et al. (2008) a 21 familias, 53 géneros, 130 especies y diez variedades (Cuadro 1 y Apéndice 1). Sólo nueve de las especies incluidas en el Apéndice 1, son producto de la revisión de ejemplares en los herbarios: Ctenitis hemsleyana, Elaphoglossum guatemalense, E. petiolatum, E. seminudum, Lycopodium clavatum, L. thyoides, Polypodium polypodioides var. interjecta, $P$. subpetiolatum y Pteris pulchra. Considerando la distribución de las especies que se señalan en la obra de Mickel y Smith (2004) y en trabajos recientes para el estado de Hidalgo (Ramírez-Cruz et al., 2009; Ceja-Romero et al., 2010; Pérez-Paredes et al., 2012), se encontró que una de las especies de licopodios y 11 de las de helechos en el presente estudio, representan nuevos registros para el estado de Hidalgo. Además, es importante mencionar que cinco de las especies presentes en el BMM

Cuadro 1. Número de familias, géneros y especies de Pteridofitas en el BMM del municipio de Tlanchinol. *Pérez-Paredes et al. (2011).

\begin{tabular}{lccc}
\hline & Familias & Géneros & Especies \\
\hline Polypodiophyta & 19 & 49 & 115 \\
Licopodiophyta & 2 & 4 & 15 \\
Total de taxones & 21 & 53 & 130 \\
$\begin{array}{l}\text { Porcentaje con respecto al estado } \\
\text { de Hidalgo* }\end{array}$ & $70 \%$ & $66.25 \%$ & $35.91 \%$ \\
\hline
\end{tabular}

Cuadro 2. Número de géneros y especies por familia de helechos y licopodios del municipio de Tlanchinol, Hidalgo. *Familias de licopodios

\begin{tabular}{|c|c|c|}
\hline Familia & Géneros & Especies \\
\hline Pteridaceae & 10 & 19 \\
\hline Polypodiaceae & 7 & 22 \\
\hline Dryopteridaceae & 6 & 20 \\
\hline Dennstaedtiaceae & 3 & 5 \\
\hline Gleicheniaceae & 3 & 3 \\
\hline Blechnaceae & 2 & 6 \\
\hline Cyatheaceae & 2 & 2 \\
\hline Dicksoniaceae & 2 & 2 \\
\hline Hymenophyllaceae & 2 & 7 \\
\hline Lycopodiaceae * & 3 & 4 \\
\hline Thelypteridaceae & 2 & 8 \\
\hline Woodsiaceae & 2 & 4 \\
\hline Aspleniaceae & 1 & 10 \\
\hline Anemiaceae & 1 & 1 \\
\hline Equisetaceae & 1 & 1 \\
\hline Marattiaceae & 1 & 1 \\
\hline Ophioglossaceae & 1 & 1 \\
\hline Plagiogyriaceae & 1 & 1 \\
\hline Psilotaceae & 1 & 1 \\
\hline Selaginellaceae * & 1 & 11 \\
\hline Tectariaceae & 1 & 1 \\
\hline
\end{tabular}

del municipio de Tlanchinol, están incluidas en alguna categoría de riesgo dentro de la Norma Oficial Mexicana NOM059-SEMARNAT-2010 (SEMARNAT, 2010).

De las 21 familias de helechos y licopodios registradas, las que poseen mayor número de géneros son Pteridaceae con diez, Polypodiaceae con siete, Dryopteridaceae con seis, y Dennstaedtiaceae y Gleicheniaceae con tres. Las familias con mayor número de especies fueron Polypodiaceae (22), Dryopteridaceae (20), Pteridaceae (19), Selaginellaceae (11) y Aspleniaceae (10) (Cuadro 2). Los géneros con mayor número de especies fueron Polypodium y Selaginella (11), Asplenium (10), Elaphoglossum (9), Thelypteris (7), Adiantum (5), Pteris (5), Trichomanes (4), Ctenitis (4), Campyloneurum (3) y Blechnum (3). Los sustratos más comunes en los que se recolectaron los ejemplares de helechos y licopodios fueron: sobre suelo (58.1\%), epífitos $(25.5 \%)$, rupícolas $(16.3 \%)$ y sobre troncos y ramas caídas $(2.4 \%)$.

Las especies con más amplia distribución en el municipio de Tlanchinol fueron Asplenium auriculatum y Pteris orizabae observadas en $63 \%$ de las localidades, Asplenium monanthes en $60 \%$, Polypodium plebeium en 53\%, Pecluma alfredii var. cupreolepis en 46\%, Elaphoglossum obscurum y E. sartorii en $40 \%$, Trichomanes radicans en $36 \%$ y Campyloneurum xalapense en $33 \%$.

En las parcelas de muestreo sólo se recolectaron 78 especies, dentro de las cuales destacan las que además de tener amplia distribución, contienen un número elevado de indivi- 
Cuadro 3. Índice de biodiversidad taxonómica (IB) en diferentes regiones del estado de Hidalgo donde se han realizado inventarios exclusivamente con Pteridofitas. Se incluyen datos del intervalo de altitud de cada área natural protegida y de cada municipio con bosque mesofilo de montaña. $\mathrm{PNC}=$ Parque Nacional El Chico; PNM = Parque Nacional los Mármoles; RBBM = Reserva de la Biosfera Barranca de Metztitlán

\begin{tabular}{lcccc}
\hline Localidad & Altitud (m) & Área (ha) & $\begin{array}{c}\text { No. de } \\
\text { especies }\end{array}$ & IB \\
\hline PNC, Hidalgo & $2,282-2,982$ & 2,739 & 62 & 7.83 \\
PNM, Hidalgo & $1,300-2,950$ & 23,150 & 71 & 6.97 \\
RBBM, Hidalgo & $1,000-2,000$ & 96,000 & 79 & 6.89 \\
Calnali, Hidalgo & $318-2,370$ & 19,020 & 115 & 11.67 \\
$\quad$ Tenango de Doria, & $630-2,250$ & 21,070 & 111 & 11.15 \\
$\quad$ Hidalgo & & & & \\
Tlanchinol, Hidalgo & $1,107-1,900$ & 38,000 & 130 & 12.33 \\
Zacualtipán, Hidalgo & $1,004-2,050$ & 24,160 & 125 & 12.39 \\
\hline
\end{tabular}

duos por unidad de área: Asplenium sessilifolium var. sessilifolium, Elaphoglossum obscurum, E. sartorii, Pecluma alfredii var. cupreolepis y Polypodium plebeium (Apéndice 1).

Los estimadores de la riqueza de especies utilizados, sugieren porcentajes satisfactorios de completitud del inventario. El estimador ACE indica que la riqueza podría incrementarse hasta 149 especies ( $87 \%$ de completitud), mientras que el modelo de Clench estimó que el número de especies podría ser hasta de 177 (73.4\% de completitud).

Los valores del índice de biodiversidad taxonómica para los BMM de los municipios de Calnali, Tenango de Doria, Zacualtipán de Ángeles y Tlanchinol fluctuaron entre 11.15 y 12.39. En cambio en las áreas naturales protegidas (PNC, PNM y RBBM), donde las condiciones ambientales son menos benignas (menor precipitación pluvial, temperatura promedio anual elevada) y que poseen tipos de vegetación diferentes, los valores de IB fueron menores de ocho (Cuadro 3).

\section{Discusión}

La lista de helechos y licopodios documentada por LunaVega et al. (1994) para el BMM del municipio de Tlanchinol fue de 15 familias, 28 géneros y 43 especies. Sin embargo, en el presente estudio se encontró que la riqueza de taxones es considerablemente mayor con 21 familias, 53 géneros, 130 especies y diez variedades; además de que 12 de las especies identificadas representan nuevos registros para el estado de Hidalgo.

Es importante mencionar que aunque el inventario florístico del presente estudio versó exclusivamente sobre Pteridofitas, no se encontraron en campo, ni en la revisión de ejemplares en los herbarios, cinco de las especies registradas por Luna-Vega et al. (1994): Asplenium alatum Humb. \& Bonpl. ex Willd., Campyloneurum tenuipes Maxon, Cyathea aff. divergens Kunze, Polypodium sanctae-rosae
(Maxon) C.Chr. y Pteris aff. propinqua J.Agardh; y 19 de las especies incluidas en un estudio previo realizado por Franco-Hernández (2005): Adiantum tenerum Sw., Anogramma leptophylla (L.) Link, Cheilanthes angustifolia Kunth, C. bonariensis (Willd.) Proctor, Cystopteris membranifolia Mickel, Dennstaedtia globulifera (Poir.) Hieron., Hypolepis blepharochlaena Mickel \& Beitel, Mildella intramarginalis (Kaulf. ex Link) Trevis., Pecluma dispersa (A.M.Evans) M.G.Price, Phanerophlebia nobilis (Schltdl. \& Cham.) C. Presl, Pityrogramma trifoliata (L.) R.M.Tryon, Polypodium conterminans Liebm., Polypodium hispidulum Bartlett, Pteridium aquilinum (L.) Kuhn in v.d. Decken var. feei (W.Schaffn. ex Féé) Maxon ex Yunck., Sticherus bifidus (Willd.) Ching, Sticherus palmatus (W.Schaffn. ex E.Fourn.) Copel., Thelypteris cinerea (Sodiro) A.R.Sm., Thelypteris resinífera (Desv.) Proctor y Woodsia mollis (Kaulf.) J.Sm.

La consulta de ejemplares en los herbarios, permitió confirmar la presencia en el municipio de sólo seis de las especies mencionadas en los estudios previos. Con base en los resultados del trabajo en campo y de la revisión de herbarios, el BMM de Tlanchinol alberga 130 especies de helechos y licopodios, cifra que lo convierte en el municipio con mayor riqueza de especies de Pteridofitas del estado de Hidalgo (Pérez-Paredes et al., 2011, 2012). Aun así, los dos estimadores de riqueza de especies utilizados indicaron que es necesario un mayor esfuerzo de recolecta para mejorar la completitud del inventario de la Pteridoflora del municipio de Tlanchinol. Por ello, es importante considerar que la completitud del inventario se incrementaría hasta $87 \%$ con el modelo de Clench y hasta $100 \%$ con el estimador ACE, si se lograra añadir los 24 taxones que se identificaron en los estudios de Luna-Vega et al. (1994) y Franco-Hernández (2005). Sin embargo, su presencia en el municipio sólo podrá corroborarse con trabajo de campo adicional y/o cuando los ejemplares botánicos de referencia sean depositados en los herbarios.

Nakamura y Soberón (2008) mencionan que la detección de especies para "completar" un inventario, depende directamente de su abundancia, del método de muestreo y de la experiencia del investigador. En el presente estudio se determinó que $81 \%$ de las especies de helechos y licopodios poseía una densidad promedio baja por unidad de área (menos de cinco individuos $/ 400 \mathrm{~m}^{2}$ ); estos valores podrían afectar la estimación de los valores de riqueza esperada (Jiménez-Valverde y Hortal, 2003; González-Oreja et al., 2010), particularmente con el modelo de Clench, donde la riqueza esperada se estimó en 177 especies. Este modelo predice que, conforme el inventario se va completando, se requiere de un esfuerzo de muestreo cada vez mayor para encontrar más especies (López-Gómez y Williams-Linera, 2006). Considerado los patrones de abundancia de los helechos y licopodios en el BMM de Tlanchinol (pocas especies abundantes y muchas especies raras) y los comentarios de Jiménez-Valverde y Hortal (2003) y González-Oreja et al. 
(2010) para el modelo Clench, un mayor esfuerzo de muestreo aportaría realmente pocas especies al inventario y éstas serían escasas a nivel local o bien, podrían estar representadas por individuos provenientes de localidades o tipos de vegetación contiguos.

Es de llamar la atención, por la cantidad de trabajos y visitas de botánicos que ha habido en el municipio estudiado, que 12 de las especies de helechos y licopodios sean nuevos registros para Hidalgo; ocho son de amplia distribución en México y se encuentran en los estados colindantes. En cambio la distribución de las otras cuatro especies (Asplenium barbaense, A. tuerckheimii, Hymenophyllum ectocarpon y Megalastrum atrogriseum) se restringe a tres o menos entidades (Mickel y Smith, 2004), lo que probablemente se deba a la escasez de inventarios con Pteridofitas en otros estados de la República Mexicana.

Los estudios recientes sobre la riqueza de especies de helechos y licopodios en Hidalgo han aportado resultados sobresalientes; en el inventario de la Pteridoflora de la RBBM, Cuevas-Hernández (2008) menciona cinco nuevos registros, en el PNM Ramírez-Cruz et al. (2009) encontraron siete, y en el municipio de Zacualtipán de Ángeles se mencionan cuatro (Pérez-Paredes et al., 2012). Considerando esta información y los 12 nuevos registros del presente estudio, la riqueza de Pteridofitas conocida para el estado de Hidalgo asciende hasta el momento a 362 especies.

Los géneros con mayor número de especies en el BMM de Tlanchinol (Asplenium, Elaphoglossum, Polypodium, Selaginella y Thelypteris) presentaron también alta riqueza en otros municipios de la entidad con el mismo tipo de vegetación (Pérez-Cervantes, 2009; Zúñiga-Salvatierra, 2009; Pérez-Paredes et al., 2012). Estos cinco géneros representan el $37.2 \%$ del total de las especies en el área de estudio, por lo que constituyen una referente más para la caracterización del BMM; se reconoce que Asplenium, Elaphoglossum y Polypodium son propios de las zonas templadas de montaña, mientras que Thelypteris y Selaginella lo son de zonas más cálidas o tropicales (Tejero-Díez y Arreguín Sánchez, 2004; Tejero-Díez et al., 2011).

El tipo de sustrato más común en el que se desarrollan los helechos y licopodios en el municipio de Tlanchinol fue el terrestre y después el epífito. Este resultado se debe principalmente a que son las dos formas de vida que predominan en las Pteridofitas a nivel mundial (Watkins et al., 2006). En México, el sustrato de crecimiento más común es el terrestre (Pérez-García et al., 1995); sin embargo, la forma de vida epifita en los helechos se incrementa en el ecosistema templado húmedo de montaña, de ahí su alta proporción en el BMM de Tlanchinol con respecto a otros tipos de vegetación (Rzedowski, 1978; Pérez-García et al., 1995; RamírezCruz et al., 2009; Tejero-Díez et al., 2011; Pérez-Paredes et al., 2012).

En la relación especies-área, se considera que independientemente del grupo taxonómico o del tipo de ecosistema de que se trate, el número de especies tiende a incrementarse conforme aumenta el tamaño del área (Whittaker et al., 2001). Sin embargo, existen varios factores independientes de la relación especies/área, que son igualmente críticos y tienen también un alto poder explicativo de los patrones de riqueza de especies, tales como la productividad, la disponibilidad de energía (clima) y la heterogeneidad ambiental (Whittaker et al., 2001; Triantis et al., 2008). En el caso del BMM de Tlanchinol, el valor del índice de diversidad taxonómica fue de 12.33 especies de helechos y licopodios por ha, valor similar al que poseen los BMM de Calnali, Tenango de Doria y Zacualtipan de Ángeles (Pérez-Cervantes, 2009; Zúñiga-Salvatierra, 2009; Pérez-Paredes et al., 2012), lo que se relaciona con la semejanza en las condiciones ambientales (clima templado húmedo) y fisiográficas que predominan en los cuatro municipios (Cuadro 3), idóneas para el desarrollo de las Pteridofitas (Moran, 2004; Tejero-Díez y Arreguín-Sánchez, 2004; Watkins et al., 2006; RamírezCruz et al., 2009; Sharpe et al., 2010; Tejero-Díez et al., 2011). Además de que está bien documentado que el BMM es el tipo de vegetación que posee la más elevada riqueza florística por unidad de área de todos los tipos de vegetación de México (Rzedowski, 1978; Villaseñor, 2010).

Por el contrario, en regiones con condiciones ambientales menos benignas para las Pteridofitas (menor humedad), como la RBBM, con climas que varían de seco-semicálidos a semiárido templados y en donde predomina el matorral xerófilo (Cuevas-Hernández, 2008); en el PNC y en el PNM, con clima templado-subhúmedo, donde se presentan bosques de coníferas y de encino (Ramírez-Cruz et al., 2009; Serrano-Martínez, 2010), la diversidad taxonómica fue menor que en los BMM de la entidad.

Los resultados de estudios previos realizados en los BMM del estado de Hidalgo, sugerían que la riqueza de especies de helechos y licopodios era baja (Luna-Vega et al., 1994; Alcántara-Ayala y Luna-Vega, 1997; Alcántara-Ayala y Luna-Vega, 2001; Ponce-Vargas et al., 2006). Sin embargo, cuando los inventarios se realizan exclusivamente con Pteridofitas, el esfuerzo de muestreo es semejante y se refleja en resultados más confiables, menos contrastantes y susceptibles de comparación. Por ejemplo, el número de especies mencionadas recientemente por Pérez-Cervantes (2009) en el municipio de Calnali (115 especies), Zúñiga-Salvatierra (2009), en el municipio de Tenango de Doria (107 especies), Pérez-Paredes et al. (2012), en el municipio de Zacualtipán de Ángeles (126 especies) y en el presente trabajo (130 especies), permiten aseverar que los BMM de la entidad contienen una elevada riqueza de especies, comparable a la de otros municipios de México con BMM, como el de La Banderilla, Veracruz $\left(22.21 \mathrm{~km}^{2}\right)$, donde se encontraron 130 especies de Pteridofitas (Vázquez et al., 2006), lo que se puede atribuir principalmente a la semejanza en las condiciones ambientales (tipo de vegetación, fisiografía y clima) y en menor medida a sesgos en el esfuerzo de muestreo. 


\section{LAS PTERIDOFITAS DE TLANCHINOL}

Los BMM en el estado de Hidalgo no cuentan con planes de conservación y manejo, lo cual está provocando su deterioro a un ritmo alarmante (Luna-Vega et al., 2000). En el caso específico del BMM del municipio de Tlanchinol, es urgente su protección, ya que posee un porcentaje elevado (35.9\%) de las especies de helechos y licopodios presentes en la entidad; y cinco de las especies identificadas se encuentran en alguna categoría de riesgo en la NOM-059SEMARNAT-2010 (SEMARNAT, 2010): Alsophila firma, Cyathea fulva, Dicksonia sellowiana, Marattia weinmanniifolia (sujetas a protección especial) y Psilotum complanatum (amenazada). La información generada en el presente estudio, puede servir de referencia en los programas de conservación y manejo: por su importancia estructural en el sotobosque, un cambio evidente en la composición y la abundancia de las especies de helechos y licopodios en el BMM de Tlanchinol, puede ser un indicador de alteraciones en las condiciones ambientales, y por ende, en la calidad del hábitat (Salovaara et al., 2004; Cárdenas et al., 2007; Sharpe et al., 2010).

\section{Agradecimientos}

Al Consejo Nacional de Ciencia y Tecnología (CONACYT), por la beca de posgrado (259522) otorgada a la primera autora. Agradecemos a Juan Reyes Rivera por su ayuda en el trabajo de campo y a Ernesto Chanes Rodríguez por su apoyo en la revisión de los ejemplares en el herbario XAL. Esta investigación contó con apoyo económico para el trabajo en campo del proyecto FOMIX-Hidalgo, clave 95828 titulado "Diversidad Biológica del estado de Hidalgo" (segunda fase). Agradecemos los comentarios y sugerencias de Victoria Hernández-Hernández y de un(a) revisor(a) anónimo(a), los cuales contribuyeron a mejorar substancialmente el presente artículo.

\section{Literatura citada}

Alcántara-Ayala O. y Luna-Vega I. 1997. Florística y análisis biogeográfico del bosque mesófilo de montaña de Tenango de Doria, Hidalgo, México. Anales del Instituto de Biología de la Universidad Nacional Autónoma de México, Serie Botánica 68:57-106.

Alcántara-Ayala O. y Luna-Vega I. 2001. Análisis florísticos de dos áreas con bosque mesófilo de montaña en el estado de Hidalgo, México: Eloxochitlán y Tlahuelompa. Acta Botanica Mexicana 54:51-87.

Cárdenas G.G., Halme J.K. y Tuomisto H. 2007. Riqueza y distribución ecológica de especies de Pteridofitas en la zona del río Yavarí-Mirín, Amazonía Peruana. Biotropica 39:637-646.

Ceja-Romero J., Mendoza-Ruiz A., López-Ferrari A.R., EspejoSerna A., Pérez-García B. y García-Cruz J. 2010. Las epífitas vasculares del estado de Hidalgo, México: diversidad y distribución. Acta Botanica Mexicana 93:1-39.

Colwell R. 2009. EstimateS, v8.2 Statistical estimation of species richness and shared species from samples. University of Con- necticut, Connecticut. Disponible en línea: <viceroy.eeb.uconn. edu/EstimateS $>$ (consultada el 2 de junio de 2011).

Cuevas-Hernández H.A.L. 2008. Los helechos y licopodios de la Reserva de la Biosfera Barranca de Metztitlán, estado de Hidalgo, México. Tesis de Licenciatura, Universidad Autónoma del Estado de Hidalgo. Hidalgo. 58 pp.

Franco-Hernández P.F. 2005. Pteridoflora del bosque mesófilo de montaña de Tlanchinol, Hidalgo, México. Tesis de Licenciatura. Instituto Tecnológico Agropecuario $\mathrm{N}^{\circ} 6$. Huejutla, Hidalgo . $315 \mathrm{pp}$.

González-Oreja J.A., de la Fuente-Díaz-Ordaz A.A., HernándezSantín L., Buzo-Franco D. y Bonache-Regidor C. 2010. Evaluación de estimadores no paramétricos de la riqueza de especies. Un ejemplo con aves en áreas verdes de la ciudad de Puebla, México. Animal Biodiversity and Conservation 33:31-45.

INEGI [Instituto Nacional de Estadística Geografía e Informática]. 1992. Síntesis geográfica del estado de Hidalgo. INEGI, Aguascalientes.

INEGI. 1996. Tlanchinol, estado de Hidalgo. INEGI, Aguascalientes.

INEGI. 2004. Anuario estadístico. Hidalgo. I, II. INEGI, Aguascalientes.

Jiménez-Valverde A. y Hortal J. 2003. Las curvas de acumulación de especies y la necesidad de evaluar la calidad de los inventarios biológicos. Revista Ibérica de Aracnología 8:151-161.

Kessler M. 2002. Range size and its ecological correlates among the pteridophytes of Carrasco National Park, Bolivia. Global Ecology and Biogeography 11:89-102.

Kessler M. 2010. Biogeography of ferns. En: Mehltreter K., Walker L.R. y Sharpe J.M. Eds. Fern Ecology, pp. 22-60, Cambridge University Press, Nueva York.

León y Paniagua L., Luna-Vega I., Martínez-Morales M.A. y Tejero-Díez J. D. 2010. Huasteca Alta Hidalguense. En: CONABIO [Comisión Nacional para el Conocimiento y Uso de la Biodiversidad] Comp. El Bosque Mesófilo de Montaña en México: Amenazas y Oportunidades para su Conservación y Manejo Sostenible, pp. 60-63, CONABIO, México, D.F.

López-Gómez A.M. y Williams-Linera G. 2006. Evaluación de métodos no paramétricos para la estimación de la riqueza de especies de plantas leñosas en cafetales. Boletín de la Sociedad Botánica de México 78:7-15.

Luna-Vega I., Ocegueda-Cruz S. y Alcántara-Ayala O. 1994. Florística y notas biogeográficas del bosque mesófilo de montaña del municipio de Tlanchinol, Hidalgo, México. Anales del Instituto de Biología de la Universidad Nacional Autónoma de México, Serie Botánica 65:31-62.

Luna-Vega I., Alcántara-Ayala O., Morrone J.J. y Espinosa-Organista D. 2000. Track analysis and conservation priorities in the cloud forests of Hidalgo, Mexico. Diversity and Distributions 6:137-143.

Magurran A.E. 2004. Measuring Biological Diversity. Blackwell Publishing, Malden.

Mayorga-Saucedo R., Luna-Vega I. y Alcántara-Ayala O. 1998. Florística del bosque mesófilo de montaña de Molocotlán, Molango-Xochicoatlán, Hidalgo, México. Boletín de la Sociedad Botánica de México 63:101-119.

Mehltreter K. 2008. Phenology and habitat specificity of tropical ferns. En: Ranker T.A. y Haufler C.H. Eds. Biology and Evolution of Ferns and Lycophytes, pp. 201-221, Cambridge University Press, Nueva York.

Mickel J.T. y Smith A.R. 2004. The Pteridophytes of Mexico. Me- 
moirs of the New York Botanical Garden. New York Botanical Press, Nueva York.

Moran R.C. 2004. A Natural History of Ferns. Timber Press, Portland.

Nakamura M. y Soberón J. 2008. Use of approximate inference in an index of completeness of biological inventories. Conservation Biology 23:469-474.

Ortega-Escalona F. y Castillo-Campos G. 1996. El bosque mesófilo de montaña y su importancia forestal. Ciencias 43:32-39.

Pavón-Hernández N.P. y Meza-Sánchez M. 2009. Cambio Climático en el Estado de Hidalgo: Clasificación y Tendencias Climáticas. Universidad Autónoma del Estado de Hidalgo, Pachuca.

Pérez-García B., Riba R. y Reyes-Jaramillo I. 1995. Helechos mexicanos: formas de crecimiento, hábitat y variantes edáficas. Contactos 11:22-27.

Pérez-Cervantes A. 2009. Helechos y licopodios del municipio de Calnali, estado de Hidalgo, México. Tesis de Licenciatura, Universidad Autónoma del Estado de Hidalgo. Hidalgo. 97 pp.

Pérez-Paredes M.G., Sánchez-González A. y Tejero-Díez J.D. 2012. Listado de licopodios y helechos del municipio de Zacualtipán de Ángeles, Hidalgo, México. Polibotánica 33:57-73.

Pérez-Paredes M.G., Serrano-Martínez H., Álvarez-Zúñiga E. y Sánchez-González A. 2011. Riqueza de especies de helechos y licopodios en el estado de Hidalgo. Herreriana, Revista de Divulgación de la Ciencia 7:5-7.

Ponce M., Mehltreter K. y de la Sota E.R. 2002. Análisis biogeográfico de la diversidad pteridofítica en Argentina y Chile continental. Revista Chilena de Historia Natural 75:703-717.

Ponce-Vargas A., Luna-Vega I., Alcántara-Ayala O. y Ruiz-Jiménez C.A. 2006. Florística del bosque mesófilo de montaña de Monte Grande, Lolotla, Hidalgo, México. Revista Mexicana de Biodiversidad 77:1177-1190.

Puig H. 1976. Végétation de la Huasteca, Mexique. Mission Archeologique et Ethnologique Française au Mexique. México, D.F.

Ramírez-Cruz S., Sánchez-González A. y Tejero-Díez D. 2009. La Pteridoflora del Parque Nacional Los Mármoles, Hidalgo, México. Boletín de la Sociedad Botánica de México 84:35-44.

Riba R. 1998. Pteridofitas mexicanas: distribución y endemismo. En: Ramamoorthy T.P., Bye R., Lot A. y Fa J. Eds. Diversidad Biológica de México. Orígenes y Distribución, pp. 369384, Instituto de Biología, Universidad Nacional Autónoma de México, México, D.F.

Rojas-Alvarado A.F. 2003. New taxa, new records and redefined concepts in the Elaphoglossum sect. Elaphoglossum subsec. Pachyglossa (Lomariopsidaceae) from Mexico and Central America. Revista de Biología Tropical 51:1-31.

Rzedowski J. 1978. Vegetación de México. Limusa, México, D.F.

Salovaara K.J., Cárdenas G.G. y Tuomisto H. 2004. Forest classification in an Amazonian rainforest landscape using pteridophytes as indicator species. Ecography 27:689-700.

Secretaría de Gobernación y Centro Estatal de Estudios Municipales de Hidalgo. 1988. Los municipios de Hidalgo. Colección: Enciclopedia de los municipios de México. Secretaría de Gobernación, México, D.F.

SEMARNAT [Secretaria del Medio Ambiente y Recursos Natura- les]. 2010. Norma Oficial Mexicana NOM-059-SEMARNAT2010, Protección ambiental - Especies nativas de México de Flora y Fauna Silvestres - Categorías de Riesgo y especificaciones para su inclusión, exclusión o cambio - Lista de Especies en Riesgo. Diario Oficial de la Federación 2a Sección, 30 de diciembre del 2010.

Serrano-Martínez H. 2010. Las Pteridofitas del Parque Nacional El Chico, Hidalgo, México. Tesis de Licenciatura en Biología. Universidad Autónoma del Estado de Hidalgo. 70 pp.

Sharpe J.M., Mehltreter K. y Walker L.R. 2010. Ecological importance of ferns. En: Mehltreter K., Walker L.R. y Sharpe J.M. Eds. Fern Ecology, pp. 1-21, Cambridge University Press, Cambridge.

Smith A.R., Pryer K.M., Schuettpelz E., Korall P., Schneider H. y Wolf P.G. 2008. Fern classification. En: Ranker T.A. y C.H. Haufler. Eds. Biology and Evolution of Ferns and Lycophytes, pp. 417-467, Cambridge University Press, Cambridge.

StatSoft, Inc. 2004. STATISTICA (data analysis software system), version 7. www.statsoft.com.

Tejero-Díez J. D. y Arreguín-Sánchez M.L. 2004 . Lista con anotaciones de los pteridófitos del estado de México, México. Acta Botanica Mexicana 69:1-82.

Tejero-Díez D., Torres-Díaz A., Mickel J.T., Mehltreter K.V.y Krömer T. 2011. Helechos y Licopodios. En: Cruz-Arango A., Lorea-Hernández F.G., Hernández-Ortiz V. y Morales-Mavil J.E. Eds. La Biodiversidad en Veracruz, Estudio de Estado Volumen 2, pp. 97-115, Comisión Nacional para el Conocimiento y Uso de la Biodiversidad/Gobierno del Estado de Veracruz/Universidad Veracruzana/Instituto de Ecología, A.C. México, D.F.

Triantis K.A., Nogués-Bravo D., Hortal J., Borges P.A.V., Adsersen H., Fernández-Palacios J.M., Araújo M.B. y Whittaker R.J. 2008. Measurements of area and the (island) species area relationship: new directions for an old pattern. Oikos 117:15551559

Vázquez-Torres M., Campos-Jiménez J. y Cruz-Pérez A. 2006. Los helechos y plantas afines del bosque mesófilo de montaña de Banderilla, Veracruz, México. Polibotánica 22:63-77.

Villaseñor J.L. 2010. El Bosque Húmedo de Montaña en México y sus Plantas Vasculares: Catálogo Florístico-Taxonómico. Comisión Nacional para el Conocimiento y Uso de la Biodiversidad, México, D.F.

Villavicencio-Nieto M.A. y Pérez-Escandón B.E. 2005. Guía de la Flora Útil de la Huasteca y la Zona Otomí-Tepehua, Hidalgo I. Universidad Autónoma del Estado de Hidalgo, Pachuca.

Watkins J.E., Cardelu C., Colwell R.K. y Moran R.C. 2006. Species richness and distribution of ferns along an elevation gradient in Costa Rica. American Journal of Botany 93:73-83.

Whittaker R.J., Willis K.J. y Field R. 2001. Scale and species richness: towards a general, hierarchical theory of species diversity. Journal of Biogeography 28:453-470.

Zúñiga-Salvatierra J.R. 2009. Los helechos y licopodios del municipio de Tenango de Doria, estado de Hidalgo, México. Tesis de Licenciatura, Universidad Autónoma del Estado de Hidalgo. Hidalgo, México. 79 pp. 


\section{LAS PTERIDOFITAS DE TLANCHINOL}

Apéndice 1. Listado de especies de helechos y licopodios del BMM del municipio de Tlanchinol, estado de Hidalgo (nomenclatura con base en Smith et al., 2008). ${ }^{*}=$ nuevos registros de especies para el estado de Hidalgo; ${ }^{* *}=$ especies encontradas en herbarios; $\mathrm{A}=$ amenazada; $\mathrm{Pr}=$ sujeta a protección especial; $\mathrm{E}=$ epífito, $\mathrm{R}=$ rupícola, $\mathrm{St}=$ sobre tronco caído, $\mathrm{T}=$ terrestre. La densidad es el promedio de 24 parcelas de muestreo, se incluye la desviación estándar; el guión indica las especies que se recolectaron fuera de las parcelas de muestreo

\begin{tabular}{lll}
\hline Categoría taxonómica & Sustrato & Densidad $/ 400 \mathrm{~m}^{2}$ \\
\hline
\end{tabular}

\section{Anemiaceae}

1. Anemia adiantifolia (L.) Sw.

T, R

$0.04 \pm 0.20$

\section{Aspleniaceae}

2. Asplenium abscissum Willd.

3. A. auriculatum Sw.

4. A. barbaense Hieron. *

5. A. blepharophorum Bertol.

6. A. cuspidatum Lam.

7. A. miradorense Liebm. *

8. A. monanthes $\mathrm{L}$.

9. A. serra Langsd. \& Fisch.

10. A. sessilifolium Desv. var. sessilifolium

11. A. tuerckheimii Maxon*

\section{Blechnaceae}

12. Blechnum appendiculatum Willd.

13. B. occidentale L.

14. B. schiedeanum Hieron.

15. Woodwardia martinezii Maxon ex Weath.

16. W. semicordata Mickel \& Beitel

17. W. spinulosa M.Martens \& Galeotti

\section{Cyatheaceae}

18. Alsophila firma (Baker) D.S.Conant $\mathrm{Pr}$ 19. Cyathea fulva Fée $\operatorname{Pr}$

\section{Dennstaedtiaceae}

20. Dennstaedtia bipinnata (Cav.) Maxon

21. D. cicutaria Hieron

22. Hypolepis repens (L.) C.Presl

23. Pteridium arachnoideum (Kaulf.) Maxon

24. P. caudatum Maxon

\section{Dicksoniaceae}

25. Dicksonia sellowiana (Pr.) Hook. $\mathrm{Pr}$ 26. Lophosoria quadripinnata C.Chr.

\section{Dryopteridaceae}

27. Arachniodes denticulata (Sw.) Ching

28. Ctenitis erinacea A.R.Sm.

29. C. equestris (Kunze) Ching var. equestris

30. C. hemsleyana (Baker ex Hemsl.) Copel. **

31. C. melanosticta (Kunze) Copel.

32. Elaphoglossum erinaceum T.Moore var. erinaceum

33. E. glaucum T.Moore

$\begin{array}{cc}\mathrm{R}, \mathrm{T} & 10.67 \pm 32.56 \\ \mathrm{E}, \mathrm{T}, \mathrm{R} & 4.25 \pm 11.32 \\ \mathrm{E}, \mathrm{R} & 0.04 \pm 0.20 \\ \mathrm{~T} & 0.04 \pm 0.20 \\ \mathrm{E}, \mathrm{R} & 3.00 \pm 14.70 \\ \mathrm{~T} & - \\ \mathrm{T}, \mathrm{R} & 3.96 \pm 14.67 \\ \mathrm{~T} & 0.08 \pm 0.41 \\ \mathrm{~T} & 23.42 \pm 66.50 \\ \mathrm{~T} & 0.37 \pm 1.84\end{array}$

$10.75 \pm 41.59$

$1.58 \pm 4.01$

$0.17 \pm 0.82$

$1.75 \pm 4.11$

$0.67 \pm 2.26$

$2.00 \pm 6.32$

$3.58 \pm 9.00$

$\mathrm{T} \quad 3.58 \pm 9.00$

$\mathrm{T}$

$1.29 \pm 3.90$

$0.79 \pm 2.47$

$0.42 \pm 1.18$

$2.00 \pm 9.18$

$0.33 \pm 1.63$

$1.79 \pm 5.40$

$0.46 \pm 2.25$

$-$

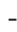

$5.21 \pm 12.34$

$1.25 \pm 6.12$

$4.46 \pm 12.90$ 
Apéndice 1. Continuación

\section{Categoría taxonómica}

34. E. guatemalense (Klotzsch) T.Moore **

35. E. obscurum (Fourn.) C.Chr.

36. E. peltatum (Sw.) Urb.

37. E. petiolatum (Sw.) Urb. **

38. E. potosianum Christ

39. E. sartorii (Liebm.) Mickel

40. E. seminudum Mickel **

41. Megalastrum atrogriseum (C.Chr.) A.R.Sm. \& R.C.Moran*

42. Phanerophlebia gastonyi Yatsk.

43. P. juglandifolia (Willd.) J.Sm.

44. P. remotispora Fourn.

45. Polystichum distans Fourn.

46. P. ordinatum Liebm.

\section{Equisetaceae}

47. Equisetum myriochaetum Schltdl. \& Cham.

\section{Gleicheniaceae}

48. Diplopterygium bancroftii (Hook.) A.R.Sm.

49. Gleichenella pectinata (Willd.) Ching

50. Sticherus underwoodianus (Maxon) Nakai

\section{Hymenophyllaceae}

51. Hymenophyllum ectocarpon Fée *

52. H. crispum Kunth

53. H. polyanthos Sw.

54. Trichomanes capillaceum L.

55. T. hymenophylloides Bosch

56. T. radicans Sw.

57. T. reptans Sw.

\section{Lycopodiaceae}

58. Huperzia reflexa (Lam.) Trevis.

59. Lycopodiella cernua (L.) Pic.Serm.

60. Lycopodium clavatum L. **

61. Lycopodium thyoides Humb. \& Bonpl. ex Willd. **

\section{Marattiaceae}

62. Marattia weinmanniifolia Liebm. $\mathrm{Pr}$

\section{Ophioglossaceae}

63. Botrychium decompositum M.Martens \& Galeotti

\section{Plagiogyriaceae}

64. Plagiogyria pectinata (Liemb.) Lellinger

\section{Polypodiaceae}

65. Campyloneurum angustifolium Fée

66. C. phyllitidis C. Presl

67. C. xalapense Fée

Sustrato

Densidad $/ 400 \mathrm{~m}^{2}$

$\begin{array}{cc}\text { E } & - \\ \text { R } & 516 \pm 1413.97 \\ \text { E, St } & 0.21 \pm 1.02 \\ \text { E, T } & - \\ \text { R } & 0.21 \pm 1.02 \\ \text { T } & 630.70 \pm 1588.89 \\ \text { T } & - \\ \text { T } & - \\ \text { T } & 3.67 \pm 7.06 \\ \text { T } & 1.33 \pm 3.86 \\ \text { T } & - \\ \text { T } & - \\ \text { T } & 1.83 \pm 7.77\end{array}$

T

T

$\mathrm{T}$

$\mathrm{T}$

$1.25 \pm 6.12$
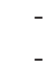

E

E

E

$\mathrm{R}$

$\mathrm{T}$

R

E, R

$1.42 \pm 4.16$

$0.04 \pm 0.20$

$5.04 \pm 14.67$

$0.13 \pm 0.61$

$0.13 \pm 0.61$

$\mathrm{T}$

$\mathrm{T}$

$\mathrm{T}$

$\mathrm{T}$

$1.67 \pm 4.20$

T

$0.92 \pm 4.49$

T

$1.96 \pm 5.49$

E, R

$4.29 \pm 10.81$

E, R

$7.38 \pm 29.50$ 
LAS PTERIDOFITAS DE TLANCHINOL

Apéndice 1. Continuación

\section{Categoría taxonómica}

Sustrato

68. Lellingeria prionodes (Mickel \& Beitel) A.R.Sm. \& R.C.Moran

69. Melpomene leptostoma (Fée) A.R.Sm. \& R.C.Moran

70. Pecluma alfredii (Rosenst.) M.G.Price var. cupreolepis (A.M.Evans) A.R.Sm.

71. P. plumula (Humb. \& Bonpl. ex Willd.) M.G.Price

72. P. sursumcurrens (Copel.) M.G.Price

73. Phlebodium pseudoaureum (Cav.) Lellinger

74. Pleopeltis crassinervata T.Moore

75. P. mexicana (Fée) Mickel \& Beitel

76a. P. polylepis T. Moore var. interjecta (Weath.) E.A.Hooper**

76b. P. polylepis T.Moore var. polylepis

77. Polypodium fraternum Cham. \& Schltdl.

78. P. hartwegianum Hook.

79. P. lepidotrichum (Fée) Maxon

80. P. longepinnulatum Fourn.

81. P. plebeium Schltdl. \& Cham.

82. P. plesiosorum Kunze

83a. P. polypodioides (L.) Watt var. aciculare Weath

83b. P. polypodioides (L.) Watt var. polypodioides

84. P. rhodopleuron Kunze

85. P. subpetiolatum Hook. in Bentham **

86. P. villagranii Copel.

\section{Psilotaceae}

87. Psilotum complanatum Sw. A

E, St

$\begin{array}{cc}\text { E } & 0.29 \pm 1.08 \\ \text { E, St } & 0.83 \pm 4.08 \\ \text { E } & 29.00 \pm 45.04 \\ \text { E, R, T } & - \\ \text { E } & - \\ \text { E, T } & 2.58 \pm 7.41 \\ \text { E } & 9.29 \pm 18.04 \\ \text { E } & 2.67 \pm 7.59 \\ \text { E } & - \\ \text { E } & 0.71 \pm 2.12 \\ \text { E } & 2.17 \pm 4.97 \\ \text { R } & - \\ \text { E } & 1.75 \pm 4.83 \\ \text { E } & 4.88 \pm 12.33 \\ \text { E, T } & 3.96 \pm 43.44 \\ \text { E, T } & 7.38 \pm 14.80 \\ \text { E } & 0.17 \pm 0.82 \\ \text { E } & 2.29 \pm 10.21 \\ \text { E } & 10.29 \pm 18.36 \\ \text { E } & - \\ \text { E } & - \\ & \end{array}$

$2.96 \pm 11.90$

\section{Pteridaceae}

88. Adiantum andicola Liebm.

89. A. braunii Mett. ex Kuhn

90. A. capillus-veneris L.

91. A. concinnum Willd. *

92. A. poiretii Wikstr.

93. Aspidotis meifolia (D.C.Eaton) Pic. Serm. *

94. Cheilanthes farinosa (Forssk.) Kaulf.

95. C. notholaenoides (Desv.) Maxon ex Weath.

96. Llavea cordifolia Lag.

97. Mildella fallax (M.Martens \& Galeotti) G.L.Nesom

98. Notholaena copelandii C.C.Hall

99. Pellaea ovata Weath.

100. Pityrogramma ebenea (L.)

101. Pteris longifolia L. *

102. P. muricella Fée*

103. P. orizabae M.Martens \& Galeotti

104. P. pulchra Schltdl. \& Cham. **

105. P. quadriaurita Retz.

106. Vittaria graminifolia Kaulf.

\section{Selaginellaceae}

107. Selaginella arsenei Weath.

108. S. extensa Underw.

109. S. flexuosa Spring
$1.46 \pm 3.24$

$1.25 \pm 6.12$

$-$

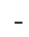

$-$

$-$

$-$

$-$

-

-

$-$

$0.04 \pm 0.20$

-

$8.63 \pm 11.64$

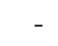

$-$

$0.92 \pm 1.89$

$0.33 \pm 1.63$
E, R

$\mathrm{E}, \mathrm{T}$ 
ERIKA ÁlvareZ-ZÚÑIGA ET AL.

Apéndice 1. Continuación

\begin{tabular}{|c|c|c|}
\hline Categoría taxonómica & Sustrato & Densidad/400 $\mathrm{m}^{2}$ \\
\hline 110. S. hoffmanni Hieron. & $\mathrm{T}$ & $1.17 \pm 2.67$ \\
\hline 111. S. lineolata Mickel \& Beitel & $\mathrm{R}, \mathrm{T}$ & $0.04 \pm 0.20$ \\
\hline 112. S. pallescens (C.Presl) Spring & $\mathrm{R}, \mathrm{T}$ & $1.21 \pm 3.44$ \\
\hline 113. S. schiedeana A.Braun* & $\mathrm{T}$ & - \\
\hline 114. S. silvestris Aspl. & $\mathrm{T}$ & $0.21 \pm 1.02$ \\
\hline 115. S. stellata Spring & $\mathrm{T}$ & - \\
\hline 116. S. tenella (P.Beauv.) Spring & $\mathrm{T}$ & - \\
\hline 117. S. wrightii Hieron. & $\mathrm{R}$ & - \\
\hline \multicolumn{3}{|l|}{ Tectariaceae } \\
\hline 118. Tectaria heracleifolia (Will.) Underw. & $\mathrm{T}$ & - \\
\hline \multicolumn{3}{|l|}{ Thelypteridaceae } \\
\hline 119. Macrothelypteris torresiana (Gaudich.) Ching & $\mathrm{T}$ & - \\
\hline 120. Thelypteris atrovirens (C.Chr.) C.F.Reed & $\mathrm{T}$ & $1.75 \pm 3.35$ \\
\hline 121. T. dentata (Forssk.) E.St.John * & $\mathrm{T}$ & $0.04 \pm 0.20$ \\
\hline 122. T. hispidula (Decne.) C.F.Reed & $\mathrm{T}$ & - \\
\hline 123. T. kunthii (Desv.) C.V.Morton & $\mathrm{T}$ & $0.04 \pm 0.20$ \\
\hline 124. T. oligocarpa (Humb. \& Bonpl. ex Willd.) Ching & $\mathrm{T}$ & $0.67 \pm 2.87$ \\
\hline 125. T. ovata R.P. St. John var. lindheimeri (C.Chr.) A.R.Sm. & $\mathrm{T}$ & - \\
\hline 126. T. puberula (Baker) C.V.Morton var. puberula & $\mathrm{T}$ & - \\
\hline \multicolumn{3}{|l|}{ Woodsiaceae } \\
\hline 127. Cystopteris fragilis (L.) Bernh. & $\mathrm{T}$ & $0.04 \pm 0.20$ \\
\hline 128. Diplazium franconis Liebm. & $\mathrm{T}$ & $0.75 \pm 2.56$ \\
\hline 129. D. lonchophyllum Kunze & $\mathrm{T}$ & $2.54 \pm 4.86$ \\
\hline 130. D. ternatum Liebm. & $\mathrm{T}$ & $1.75 \pm 7.75$ \\
\hline
\end{tabular}


LAS PTERIDOFITAS DE TLANCHINOL

Apéndice 2. Listado de especies de helechos y licopodios del bosque mesófilo de montaña del municipio de Tlanchinol, Hidalgo. La nomenclatura de los taxones se basó en Smith et al. (2008).

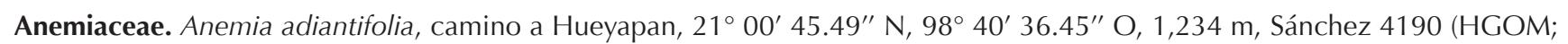
MEXU).

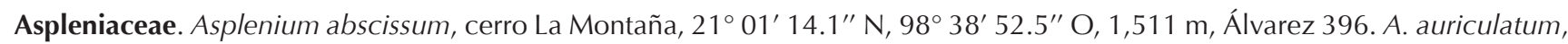
parada Lontla o rancho Temazate, $21^{\circ} 01^{\prime} 43.56^{\prime \prime} \mathrm{N}, 98^{\circ} 38^{\prime} 25.15^{\prime \prime} \mathrm{O}, 1,500 \mathrm{~m}$, Sánchez 4165. A. barbaense, La virgen (La 82), $21^{\circ} 01^{\prime} 26^{\prime \prime} \mathrm{N}, 98^{\circ} 38^{\prime} 05.0^{\prime \prime} \mathrm{O}, 1,411 \mathrm{~m}$, Álvarez 183. A. blepharophorum, terreno de Chachala, carretera a Quetzalzongo, bajo catedral, $20^{\circ} 56^{\prime} 54.9^{\prime \prime} \mathrm{N}, 98^{\circ} 41^{\prime} 19^{\prime \prime} \mathrm{O}, 1,463$ m, Sánchez 4171. A. cuspidatum, montaña el Cantil, San Cristóbal, casetas, $20^{\circ}$ $57^{\prime} 29.7^{\prime \prime} \mathrm{N}, 98^{\circ} 40^{\prime} 48.8^{\prime \prime} \mathrm{O}, 1,874 \mathrm{~m}$, Álvarez 344. A. miradorense, parada Lontla o rancho Temazate, $21^{\circ} 01^{\prime} 43.56^{\prime \prime} \mathrm{N}, 98^{\circ}$ 38' 25.15" O, 1,500 m, Sánchez 4181. A. monanthes, montaña el Cantil, San Cristóbal, casetas, $20^{\circ} 57^{\prime} 29.7^{\prime \prime} \mathrm{N}, 98^{\circ} 40^{\prime} 48.8^{\prime \prime}$ $\mathrm{O}, 1,874 \mathrm{~m}$, A. sessilifolium var. sessilifolium, El Cantil, casetas, $20^{\circ} 57^{\prime} 38^{\prime \prime} \mathrm{N}, 98^{\circ} 41^{\prime} 05.7^{\prime \prime} \mathrm{O}, 1,567 \mathrm{~m}$, Álvarez 438 . A. serra,

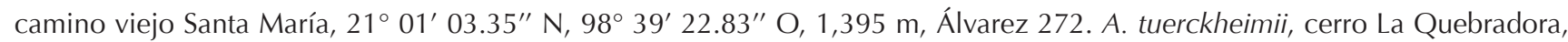
$21^{\circ} 01^{\prime} 18.7^{\prime \prime} \mathrm{N}, 98^{\circ} 36^{\prime} 35.9^{\prime \prime} \mathrm{O}, 1,273$ m, Sánchez 4193 (HGOM; MEXU).

Blechnaceae. Blechnum appendiculatum, La Virgen (La 82), 210 $01^{\prime} 26^{\prime \prime} \mathrm{N}, 98^{\circ} 38^{\prime} 05.0^{\prime \prime} \mathrm{O}, 1,411 \mathrm{~m}$, Álvarez 186. B. schiedeanum, La Virgen (La 82), $21^{\circ} 01^{\prime} 26^{\prime \prime} \mathrm{N}, 98^{\circ} 38^{\prime} 05.0^{\prime \prime} \mathrm{O}, 1,411 \mathrm{~m}$, Sánchez 4166. B. occidentale, cerro La Quebradora, $21^{\circ} 01^{\prime}$

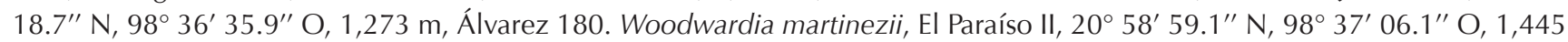
m, Álvarez 533. W. semicordata, Km. 170 por la Virgen, $21^{\circ} 01^{\prime} 25.84^{\prime \prime} \mathrm{N}, 98^{\circ} 38^{\prime} 09.62^{\prime \prime} \mathrm{O}, 1,434$ m, Álvarez 312 . W. spinulosa, Casetas, parte alta, $20^{\circ} 57^{\prime} 40.30^{\prime \prime} \mathrm{N}, 98^{\circ} 40^{\prime} 44.92^{\prime \prime} \mathrm{O}, 1,685 \mathrm{~m}$, Álvarez 468 (HGOM; MEXU).

Cyatheaceae. Alsophila firma, cerro La Quebradora, $21^{\circ} 01^{\prime} 18.7^{\prime \prime} \mathrm{N}, 98^{\circ} 36^{\prime} 35.9^{\prime \prime} \mathrm{O}, 1,273 \mathrm{~m}$, Sánchez 4175 . Cyathea fulva,

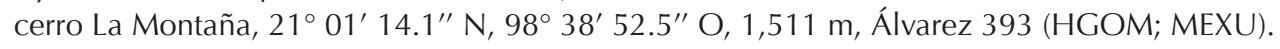

Dennstaedtiaceae. Dennstaedtia bipinnata, cerro Cholchocatipa, $21^{\circ} 03^{\prime} 20.6^{\prime \prime} \mathrm{N}, 98^{\circ} 34^{\prime} 26.2^{\prime \prime} \mathrm{O}, 1,113 \mathrm{~m}$, Álvarez 311. D. cicutaria, cerro La Quebradora, 2101' 13.1" N, 98 36' 38.4" O, 1,312 m, Álvarez 166. Hypolepis repens, camino viejo Santa María, $21^{\circ} 01^{\prime} 03.35^{\prime \prime} \mathrm{N}, 98^{\circ} 39^{\prime} 22.83^{\prime \prime} \mathrm{O}, 1,395 \mathrm{~m}$, Álvarez 281. Pteridium arachnoideum, camino a Toscalao, $20^{\circ} 58^{\prime} 43.8^{\prime \prime}$ $\mathrm{N}, 98^{\circ} 43^{\prime} 27.5^{\prime \prime} \mathrm{O}, 1,170 \mathrm{~m}$, Álvarez 537. P. caudatum, camino Ixcualamatla, $21^{\circ} 00^{\prime} 08.39^{\prime \prime} \mathrm{N}, 98^{\circ} 39^{\prime} 44.59^{\prime \prime} \mathrm{O}, 1,476 \mathrm{~m}$, Álvarez 287 (HGOM; MEXU).

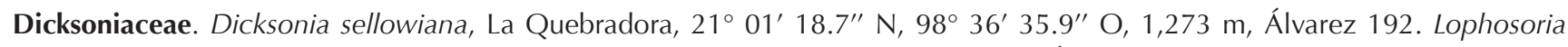
quadripinnata, camino Ixcualamatla, $21^{\circ} 00^{\prime} 08.39^{\prime \prime} \mathrm{N}, 98^{\circ} 39^{\prime} 44.59^{\prime \prime} \mathrm{O}, 1,476$ m, Álvarez 288 (HGOM; MEXU).

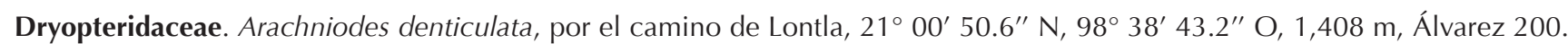
Ctenitis erinacea, El Cantil, casetas, $20^{\circ} 57^{\prime} 38^{\prime \prime} \mathrm{N}, 98^{\circ} 41^{\prime} 05.7^{\prime \prime} \mathrm{O}, 1,567 \mathrm{~m}$, Álvarez 433. C. equestris var. equestris, parada Lontla o rancho Temazate, $21^{\circ} 01^{\prime} 43.56^{\prime \prime} \mathrm{N}, 98^{\circ} 38^{\prime} 25.15^{\prime \prime} \mathrm{O}, 1,500 \mathrm{~m}$, Álvarez 226. Ctenitis hemsleyana Luna 809 (FCME). C. melanosticta, bajo catedrales, $20^{\circ} 56^{\prime} 53^{\prime \prime} \mathrm{N}, 98^{\circ} 41^{\prime} 17.1^{\prime \prime} \mathrm{O}, 1,444 \mathrm{~m}$, Álvarez 419. Elaphoglossum erinaceum var. erinaceum, Casetas, parte alta, El Cantil, $20^{\circ} 57^{\prime} 42.3^{\prime \prime} \mathrm{N}, 98^{\circ} 40^{\prime} 56.5^{\prime \prime} \mathrm{O}, 1,756 \mathrm{~m}$, Álvarez 474. E. glaucum, cerro La Montaña, sobre el arroyo, $21^{\circ} 01^{\prime} 14.1^{\prime \prime} \mathrm{N}, 98^{\circ} 38^{\prime} 52.5^{\prime \prime} \mathrm{O}, 1,511 \mathrm{~m}$, Álvarez 381. E. guatemalense, km 170-171 de la carretera federal 105, entre Tlanchinol y Tamazunchale, $20^{\circ} 58^{\prime} \mathrm{N}, 98^{\circ} 38^{\prime} \mathrm{O}, 1,200 \mathrm{~m}, 4$ de noviembre 2000, Rojas 5431 (ENCB: MEXU). E. obscurum, terreno de Chachala, carretera a Quetzalzongo, bajo catedral, $20^{\circ} 56^{\prime} 54.9^{\prime \prime} \mathrm{N}, 98^{\circ} 41^{\prime} 19^{\prime \prime} \mathrm{O}, 1,463 \mathrm{~m}$, Álvarez 412 . E. peltatum, La Bomba, 2058' 59.1" N, 98 37'06.1" O, 1,445 m, Sánchez 4158. E. petiolatum, km 170-171 de la carretera federal 105, entre Tlanchinol y Tamazunchale, $20^{\circ} 58^{\prime} \mathrm{N}, 98^{\circ} 38^{\prime} \mathrm{O}, 1,200 \mathrm{~m}, 4$ de noviembre 2000, Rojas 5432 (ENCB: MEXU). E. potosianum, montaña El Cantil, San Cristóbal, casetas, $20^{\circ} 57^{\prime} 29.9^{\prime \prime} \mathrm{N}, 98^{\circ} 40^{\prime} 55.3^{\prime \prime} \mathrm{O}, 1,836 \mathrm{~m}$, Álvarez 458. E. sartorii, montaña El Cantil, San Cristóbal, casetas, $20^{\circ} 57^{\prime}$ 29.7" N, $98^{\circ} 40^{\prime}$ 48.8” O, 1,874 m, Álvarez 338. E. seminudum, a 4 km al E de Tlanchinol, camino a Apantlasol, 3 de septiembre 1997, E. Martínez S. 28469 (MEXU). Megalastrum atrogriseum, El Cantil, casetas, 20 57' $38^{\prime \prime} \mathrm{N}, 98^{\circ} 41^{\prime}$ 05.7' O, 1,567 m, Álvarez 440. Phanerophlebia gastonyi, cerro La Quebradora, 21 $01^{\circ} 13.1^{\prime \prime} \mathrm{N}, 98^{\circ} 36^{\prime} 38.4^{\prime \prime}$ O, 1,312 m, Álvarez 141. P. juglandifolia, cerro La Quebradora, $21^{\circ} 01^{\prime} 13.1^{\prime \prime} \mathrm{N}, 98^{\circ} 36^{\prime} 38.4^{\prime \prime} \mathrm{O}, 1,312 \mathrm{~m}$, Álvarez 115 . P. remotispora, camino Ixcualamatla, $21^{\circ} 00^{\prime} 08.39^{\prime \prime} \mathrm{N}, 98^{\circ} 39^{\prime} 44.59^{\prime \prime} \mathrm{O}, 1,476 \mathrm{~m}$, Álvarez 294. Polystichum distans, por el camino de Lontla, $21^{\circ} 00^{\prime} 50.6^{\prime \prime} \mathrm{N}, 98^{\circ} 38^{\prime} 43.2^{\prime \prime} \mathrm{O}, 1,408 \mathrm{~m}$, Álvarez 214. P. ordinatum, camino viejo Santa María, $21^{\circ} 01^{\prime} 03.35^{\prime \prime} \mathrm{N}$, 9839' 22.83" O, 1,395 m, Álvarez 269 (HGOM; MEXU). 
ERIKA ÁLVAREZ-ZúNIIGA ET AL.

Apéndice 2. Continuación

Equisetaceae. Equisetum myriochaetum, arriba de Toscalao, 2058'43.8” N, 98 43' 27.5” O, 1170 m, Sánchez 4177 (HGOM; MEXU).

Gleicheniaceae. Diplopterygium bancroftii, arriba de Coatatlan, $20^{\circ} 59^{\prime} 48.3^{\prime \prime} \mathrm{N}, 98^{\circ} 36^{\prime} 39.4^{\prime \prime}$ O, 1,407 m, Álvarez 446. Gleichenella pectinata, El Paraíso II, $20^{\circ} 58^{\prime} 59.1^{\prime \prime} \mathrm{N}, 98^{\circ} 37^{\prime} 06.1^{\prime \prime} \mathrm{O}, 1,445 \mathrm{~m}$, Álvarez 525. Sticherus underwoodianus, camino a La Bomba, 2059' 5.3" N, $98^{\circ} 37^{\prime} 41.4^{\prime \prime}$ O, 1,440 m, Álvarez 442 (HGOM; MEXU).

Hymenophyllaceae. Hymenophyllum ectocarpon, casetas, parte alta, El Cantil, $20^{\circ} 57^{\prime} 42.3^{\prime \prime} \mathrm{N}, 98^{\circ} 40^{\prime} 56.5^{\prime \prime} \mathrm{O}, 1,756 \mathrm{~m}$, Álvarez 491. H. crispum, casetas, parte alta, El Cantil, $20^{\circ} 57^{\prime} 42.3^{\prime \prime} \mathrm{N}, 98^{\circ} 40^{\prime} 56.5^{\prime \prime} \mathrm{O}, 1,756 \mathrm{~m}$, Álvarez 495. H. polyanthos, cerro La Montaña, $21^{\circ} 01^{\prime} 14.1^{\prime \prime} \mathrm{N}, 98^{\circ} 38^{\prime}$ 52.5" O, 1,511 m, Álvarez 377. Trichomanes capillaceum, casetas, parte alta, El Cantil, $20^{\circ} 57^{\prime} 42.3^{\prime \prime} \mathrm{N}, 98^{\circ} 40^{\prime} 56.5^{\prime \prime} \mathrm{O}, 1,756 \mathrm{~m}$, Álvarez 498. T. hymenophylloides, cerro La Montaña, sobre el arroyo, $21^{\circ} 01^{\prime} 14.1^{\prime \prime}$

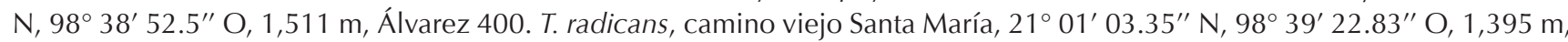
Álvarez 271. T. reptans, casetas, parte alta, el Cantil, $20^{\circ} 57^{\prime} 42.3^{\prime \prime} \mathrm{N}, 98^{\circ} 40^{\prime}$ 56.5” O, 1,756 m, Álvarez 496 (HGOM; MEXU).

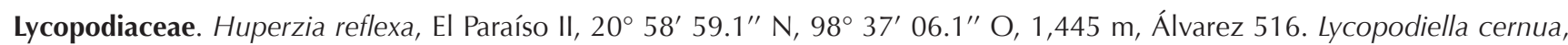
arriba de Coatatlan, 2059’ 48.3" N, 98 36' 39.4" O, 1,407 m, Sánchez 4151 (HGOM; MEXU). Lycopodium clavatum, La Virgen, 1,500 m, 29 de octubre 1994, Sabina Lara (ENCB: FCME). L. thyoides, camino a Tierra Colorada, aproximadamente a 6 km al E de Tlanchinol, 2059' 99" N, 98 36' 99" O, 1,580 m, 24 de agosto 1992, I. Luna Vega, S. Ocegueda 541 (FCME: MEXU).

Marattiaceae. Marattia weinmanniifolia, El Cantil, casetas, $20^{\circ} 57^{\prime} 38^{\prime \prime} \mathrm{N}, 98^{\circ} 41^{\prime} 05.7^{\prime \prime} \mathrm{O}, 1,567$ m, Álvarez 439 (HGOM; MEXU).

Ophioglossaceae. Botrychium decompositum, terreno de Chachala, carretera a Quetzalzongo, bajo catedral, $20^{\circ} 56^{\prime} 54.9^{\prime \prime} \mathrm{N}$, 98 41' 19"' O, 1,463 m, Álvarez 415 (HGOM; MEXU).

Plagiogyriaceae. Plagiogyria pectinata, montaña El Cantil, San Cristóbal, casetas, $20^{\circ} 57^{\prime} 29.7^{\prime \prime} \mathrm{N}, 98^{\circ} 40^{\prime} 48.8^{\prime \prime} \mathrm{O}, 1,874$ m, Álvarez 333 (HGOM; MEXU).

Polypodiaceae. Campyloneurum angustifolium, cerro La Quebradora, 21ํ 01' 13.1" N, 98 36' 38.4" O, 1,312 m, Álvarez 124. C. xalapense, cerro La Quebradora, 21 $01^{\prime} 13.1^{\prime \prime} \mathrm{N}, 98^{\circ} 36^{\prime} 38.4^{\prime \prime} \mathrm{O}, 1,312 \mathrm{~m}$, Álvarez 144. C. phyllitidis, adelante de Toscalao, $20^{\circ} 58^{\prime} 43.8^{\prime \prime} \mathrm{N}, 98^{\circ} 43^{\prime} 27.5^{\prime \prime} \mathrm{O}, 1,170 \mathrm{~m}$, Álvarez 514. Lellingeria prionodes, cerro La Montaña, sobre el arroyo, $21^{\circ} 01^{\prime} 14.1^{\prime \prime}$ $\mathrm{N}, 98^{\circ} 38^{\prime} 52.5^{\prime \prime} \mathrm{O}, 1,511 \mathrm{~m}$, Álvarez 379. Melpomene leptostoma, por el camino de Lontla, $21^{\circ} 00^{\prime} 50.6^{\prime \prime} \mathrm{N}, 98^{\circ} 38^{\prime} 43.2^{\prime \prime}$ O, 1,408 m, Álvarez 206. Pecluma alfredii var. cupreolepis, cerro La Quebradora, $21^{\circ} 01^{\prime} 13.1^{\prime \prime} \mathrm{N}, 98^{\circ} 36^{\prime} 38.4^{\prime \prime} \mathrm{O}, 1,312 \mathrm{~m}$, Álvarez 156. P. plumula, cerro La Quebradora, $21^{\circ} 01^{\prime} 13.1^{\prime \prime} \mathrm{N}, 98^{\circ} 36^{\prime} 38.4^{\prime \prime} \mathrm{O}, 1,312 \mathrm{~m}$, Álvarez 460. P. sursumcurrens, parada Lontla o rancho Temazate, $21^{\circ} 01^{\prime} 43.56^{\prime \prime} \mathrm{N}, 98^{\circ} 38^{\prime} 25.15^{\prime \prime} \mathrm{O}, 1,500 \mathrm{~m}$, Álvarez 245. Phlebodium pseudoaureum, cerro La Quebradora, $21^{\circ} 01^{\prime} 13.1^{\prime \prime} \mathrm{N}, 98^{\circ} 36^{\prime} 38.4^{\prime \prime} \mathrm{O}, 1,312 \mathrm{~m}$, Álvarez 123. Pleopeltis crassinervata, parada Lontla o rancho Temazate, $21^{\circ} 01^{\prime} 43.56^{\prime \prime} \mathrm{N}, 98^{\circ} 38^{\prime} 25.15^{\prime \prime} \mathrm{O}, 1,500 \mathrm{~m}$, Álvarez 257. P. mexicana, parada Lontla o rancho Temazate, $21^{\circ} 01^{\prime} 43.56^{\prime \prime} \mathrm{N}$,

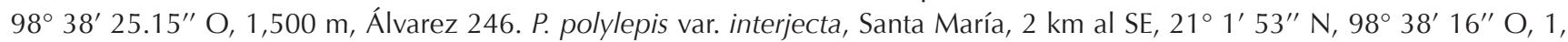
200 m, 29 de junio 1998, Rafael Mayorga Saucedo, Othón Alcántara Ayala S 1218 (FCME). P. polylepis var. polylepis, montaña El Cantil, San Cristóbal, casetas, $20^{\circ} 57^{\prime} 29.7^{\prime \prime} \mathrm{N}, 98^{\circ} 40^{\prime} 48.8^{\prime \prime} \mathrm{O}, 1.874$ m, Álvarez 342. Polypodium fraternum, bajo las catedrales, $20^{\circ} 56^{\prime} 53^{\prime \prime} \mathrm{N}, 98^{\circ} 41^{\prime} 17.1^{\prime \prime} \mathrm{O}, 1,444 \mathrm{~m}$, Sánchez 4195. P. hartwegianum, cerro La Montaña, $21^{\circ} 01^{\prime} 14.1^{\prime \prime} \mathrm{N}, 98^{\circ} 38^{\prime}$ 52.5" O, 1,511 m, Sánchez 4200. P. lepidotrichum, por el camino de Lontla, $21^{\circ} 0^{\prime} 50.6^{\prime \prime} \mathrm{N}, 98^{\circ} 38^{\prime} 43.2^{\prime \prime} \mathrm{O}, 1,408 \mathrm{~m}$, Álvarez 216. P. longepinnulatum, arriba de Coatatlan, $20^{\circ} 59^{\prime} 48.3^{\prime \prime} \mathrm{N}, 98^{\circ} 36^{\prime} 39.4^{\prime \prime} \mathrm{O}, 1,407 \mathrm{~m}$, Sánchez 4174. P. plebeium, parada Lontla o rancho Temazate, $21^{\circ} 01^{\prime} 43.56^{\prime \prime} \mathrm{N}, 98^{\circ} 38^{\prime} 25.15^{\prime \prime} \mathrm{O}, 1,500 \mathrm{~m}$, Álvarez 244. P. plesiosorum, cerro La Quebradora, $21^{\circ}$ $01^{\prime} 13.1^{\prime \prime} \mathrm{N}, 98^{\circ} 36^{\prime} 38.4^{\prime \prime} \mathrm{O}, 1,312 \mathrm{~m}$, Álvarez 153. P. polypodioides var. aciculare, camino Ixcualamatla, $21^{\circ} 00^{\prime} 08.39^{\prime \prime} \mathrm{N}$, 9839' 44.59" O, 1,476 m, Sánchez 4168. P. polypodioides var. polypodioides, cerro La Quebradora, $21^{\circ} 01^{\prime} 13.1^{\prime \prime} \mathrm{N}, 98^{\circ} 36^{\prime}$ 38.4" O, 1,312 m, Álvarez 164. P. rhodopleuron, parada Lontla o rancho Temazate, $21^{\circ} 01^{\prime} 43.56^{\prime \prime} \mathrm{N}, 98^{\circ} 38^{\prime} 25.15^{\prime \prime} \mathrm{O}, 1,500$ m, Álvarez 224. P. subpetiolatum, las norias, 2 km al oeste de Tlanchinol, 1,500 m, 12 de septiembre 2000, Claudia Hernández 462. P. villagranii, cerro Cholchocatipa, $21^{\circ} 03^{\prime} 20.6^{\prime \prime} \mathrm{N}, 98^{\circ} 34^{\prime} 26.2^{\prime \prime} \mathrm{O}, 1,113 \mathrm{~m}$, Álvarez 303. (HGOM; MEXU).

Psilotaceae. Psilotum complanatum, por el camino de Lontla, $21^{\circ} 0^{\prime} 50.6^{\prime \prime} \mathrm{N}, 98^{\circ} 38^{\prime} 43.2^{\prime \prime} \mathrm{O}, 1$,408 m, Álvarez 197 (HGOM; MEXU), 
LAS PTERIDOFITAS DE TLANCHINOL

Apéndice 2. Continuación

Pteridaceae. Adiantum andicola, bajo catedrales, cerro Quetzalzongo, 2056'47.98” N, 98 41' 20.96" O, 1,388 m, Álvarez 365. A. braunii, camino Ixcualamatla, $21^{\circ} 00^{\prime} 035^{\prime \prime} \mathrm{N}, 98^{\circ} 39^{\prime} 39.5^{\prime \prime} \mathrm{O}, 1,476 \mathrm{~m}$, Álvarez 296. A. capillus-veneris, parada La Virgen, entrada a San Cristóbal, $20^{\circ} 57^{\prime} 55.1^{\prime \prime} \mathrm{N}, 98^{\circ} 40^{\prime} 23^{\prime \prime} \mathrm{O}, 1,440 \mathrm{~m}$, Álvarez 372. A. poiretii, casetas, parte alta, $20^{\circ} 57^{\prime}$

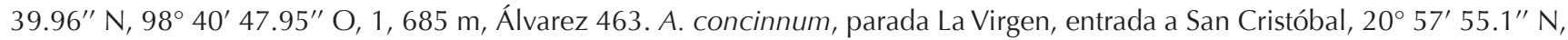
$98^{\circ} 40^{\prime} 23^{\prime \prime} \mathrm{O}, 1,440 \mathrm{~m}$, Álvarez 367. Aspidotis meifolia, bajo catedrales, cerro Quetzalzongo, $20^{\circ} 56^{\prime} 47.98^{\prime \prime} \mathrm{N}, 98^{\circ} 41^{\prime} 20.96^{\prime \prime}$ O, 1,388 m, Álvarez 366. Cheilanthes farinosa, cerro Cholchocatipa, $21^{\circ} 03^{\prime} 20.6^{\prime \prime} \mathrm{N}, 98^{\circ} 34^{\prime} 26.2^{\prime \prime} \mathrm{O}, 1$, $113 \mathrm{~m}$, Álvarez 318 . C. notholaenoides, arriba de Coatatlan, $20^{\circ} 59^{\prime} 48.4^{\prime \prime} \mathrm{N}, 98^{\circ} 36^{\prime} 41.9^{\prime \prime} \mathrm{O}, 1,406 \mathrm{~m}$, Álvarez 534. Llavea cordifolia, La Quebradora, $21^{\circ} 01^{\prime} 13.1^{\prime \prime} \mathrm{N}, 98^{\circ} 36^{\prime} 38.4^{\prime \prime} \mathrm{O}, 1,110 \mathrm{~m}$, Sánchez 4173. Mildella fallax, parada Lontla o rancho Temazate, $21^{\circ} 01^{\prime} 43.56^{\prime \prime} \mathrm{N}$,

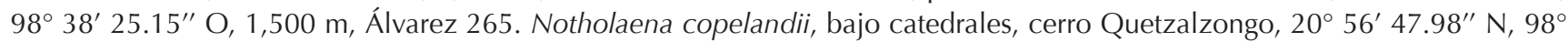

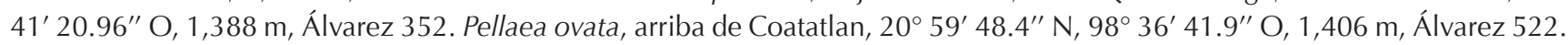

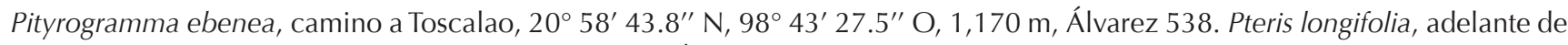
Toscalao, $20^{\circ} 58^{\prime} 43.8^{\prime \prime} \mathrm{N}, 98^{\circ} 43^{\prime} 27.5^{\prime \prime} \mathrm{O}, 1,170 \mathrm{~m}$, Álvarez 518. P. muricella, La Quebradora, $21^{\circ} 01^{\prime} 13.1^{\prime \prime} \mathrm{N}, 98^{\circ} 36^{\prime} 38.4^{\prime \prime}$ O, 1,110 m, Álvarez 511. P. orizabae, La Quebradora, parte baja, $21^{\circ} 01^{\prime} 27.9^{\prime \prime} \mathrm{N}, 98^{\circ} 36^{\prime} 39.3^{\prime \prime} \mathrm{O}, 1$, 107 m, Álvarez 403. .. pulchra, Chipoco, cerca de Otongo, 1,300 m, 1 de noviembre 1974, Rzedowski 32476 (ENCB). P. quadriaurita, arriba de Toscalao, $20^{\circ} 58^{\prime} 43.8^{\prime \prime} \mathrm{N}, 98^{\circ} 43^{\prime} 27.5^{\prime \prime} \mathrm{O}, 1,170 \mathrm{~m}$, Álvarez 517. Vittaria graminifolia, montaña El Cantil, San Cristóbal, casetas, $20^{\circ}$ $57^{\prime} 29.7^{\prime \prime} \mathrm{N}, 98^{\circ} 40^{\prime} 48.8^{\prime \prime} \mathrm{O}, 1,874$ m, Álvarez 348 (HGOM; MEXU).

Selaginellaceae. Selaginella arsenei, camino Ixcualamatla, $21^{\circ} 00^{\prime} 08.39^{\prime \prime} \mathrm{N}, 98^{\circ} 39^{\prime} 44.59^{\prime \prime} \mathrm{O}, 1,476$ m, Sánchez 4153 . S. extensa, cerro La Quebradora, 2101' $13.1^{\prime \prime} \mathrm{N}, 98^{\circ} 36^{\prime} 38.4^{\prime \prime} \mathrm{O}, 1,312 \mathrm{~m}$, Álvarez 142. S. flexuosa, Kilometro 170 por la Virgen, $21^{\circ} 01^{\prime} 4.27^{\prime \prime} \mathrm{N}, 98^{\circ} 38^{\prime} 221^{\prime \prime} \mathrm{O}, 1,434 \mathrm{~m}$, Álvarez 315. S. hoffmanni, cerro La Quebradora, $21^{\circ} 01^{\prime} 13.1^{\prime \prime} \mathrm{N}, 98^{\circ} 36^{\prime} 38.4^{\prime \prime}$ O, 1,312 m, Álvarez 502. S. lineolata, casetas, parte alta, El Cantil, $20^{\circ} 57^{\prime} 42.3^{\prime \prime} \mathrm{N}, 98^{\circ} 40^{\prime}$ 56.5” O, 1,756 m, Álvarez 489. S. $^{\prime \prime}$ pallescens, parada La Virgen, entrada a San Cristóbal, $20^{\circ} 57^{\prime} 55.1^{\prime \prime} \mathrm{N}, 98^{\circ} 40^{\prime} 23^{\prime \prime} \mathrm{O}, 1,440 \mathrm{~m}$, Álvarez 375. S. schiedeana, La Virgen (La 82), $21^{\circ} 01^{\prime} 26^{\prime \prime} \mathrm{N}, 98^{\circ} 38^{\prime} 05.0^{\prime \prime} \mathrm{O}, 1,411 \mathrm{~m}$, Álvarez 194. S. silvestris, parada de Lontla o rancho Temazate, $20^{\circ} 01^{\prime}$ 43.56" N, $98^{\circ} 38^{\prime} 25.15^{\prime \prime} \mathrm{O}, 1,500 \mathrm{~m}$, Álvarez 207. S. stellata, cerro La Quebradora, 210 $01^{\prime} 13.1^{\prime \prime} \mathrm{N}, 98^{\circ} 36^{\prime} 38.4^{\prime \prime} \mathrm{O}, 1,312$

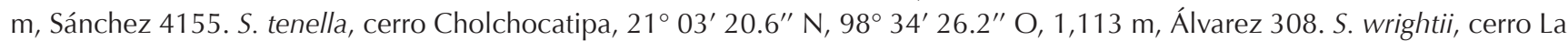
Quebradora, $21^{\circ} 01^{\prime} 21.4^{\prime \prime} \mathrm{N}, 98^{\circ} 36^{\prime} 35.2^{\prime \prime} \mathrm{O}, 1,216$ m, Sánchez 4157 (HGOM; MEXU).

Tectariaceae. Tectaria heracleifolia, camino a Toscalao, $20^{\circ} 58^{\prime} 43.8^{\prime \prime} \mathrm{N}, 98^{\circ} 43^{\prime} 27.5^{\prime \prime} \mathrm{O}, 1$,170 m, Álvarez 536 (HGOM; MEXU).

Thelypteridaceae. Macrothelypteris torresiana, arriba de Coatatlan, $20^{\circ} 59^{\prime}$ 48.4" N, 98 36' 41.9" O, 1,406 m, Sánchez 4184. Thelypteris atrovirens, arriba de Coatatlan, $20^{\circ} 59^{\prime} 48.3^{\prime \prime} \mathrm{N}, 98^{\circ} 36^{\prime} 39.4^{\prime \prime} \mathrm{O}, 1,407 \mathrm{~m}$, Sánchez 4172. T. dentata, bajo catedrales, $20^{\circ} 56^{\prime} 53^{\prime \prime} \mathrm{N}, 98^{\circ} 41^{\prime} 17.1^{\prime \prime} \mathrm{O}, 1,444 \mathrm{~m}$, Sánchez 4198. T. hispidula, cerro Cholchocatipa, 210 03' $20.6^{\prime \prime} \mathrm{N}, 98^{\circ} 34^{\prime}$ 26.2" O, 1,113 m, Sánchez 4164. T. kunthii, cajo catedrales, cerro Quetzalzongo, $20^{\circ} 56^{\prime} 47.98^{\prime \prime} \mathrm{N}, 98^{\circ} 41^{\prime} 20.96^{\prime \prime} \mathrm{O}, 1,388$ m, Álvarez 355. T. oligocarpa, Km. 170 por la Virgen, $21^{\circ} 01^{\prime} 25.84^{\prime \prime} \mathrm{N}, 98^{\circ} 38^{\prime} 09.62^{\prime \prime} \mathrm{O}, 1,434$ m, Álvarez 205. T. puberula var. puberula, cerro La Quebradora, $21^{\circ} 01^{\prime} 18.7^{\prime \prime} \mathrm{N}, 98^{\circ} 36^{\prime} 35.9^{\prime \prime} \mathrm{O}, 1,273 \mathrm{~m}$, Sánchez 4186. T. ovata var. lindheimeri, cerro La Quebradora, $21^{\circ} 01^{\prime} 13.1^{\prime \prime} \mathrm{N}, 98^{\circ} 36^{\prime} 38.4^{\prime \prime}$ O, 1,312 m, Sánchez 4180 (HGOM; MEXU).

Woodsiaceae. Cystopteris fragilis, km. 170 por la Virgen, $21^{\circ} 01^{\prime} 25.84^{\prime \prime} \mathrm{N}, 98^{\circ} 38^{\prime} 09.62^{\prime \prime} \mathrm{O}, 1,434 \mathrm{~m}$, Álvarez 316. Diplazium

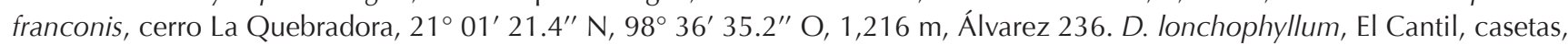
$20^{\circ} 57^{\prime} 38^{\prime \prime} \mathrm{N}, 98^{\circ} 41^{\prime} 05.7^{\prime \prime} \mathrm{O}, 1,567 \mathrm{~m}$, Álvarez 430. D. ternatum, parada Lontla o rancho Temazate, $21^{\circ} 01^{\prime} 43.56^{\prime \prime} \mathrm{N}, 98^{\circ}$ $38^{\prime} 25.15^{\prime \prime} \mathrm{O}, 1,500 \mathrm{~m}$, Álvarez 220 (HGOM; MEXU). 